\author{
Audiology \\ Neurotology
}

Audiol Neurotol 2017;22:24-29

DOI: $10.1159 / 000472245$
Received: October 18, 2016

Accepted: March 24, 2017

Published online: May 18, 2017

\title{
Delayed Effect of Active Pressure Treatment on Endolymphatic Hydrops
}

\author{
Edoardo Covelli $^{\mathrm{a}}$ Luigi Volpini $^{\mathrm{a}}$ Francesca Atturo $^{\mathrm{a}}$ Anna Teresa Benincasa ${ }^{\mathrm{a}}$ \\ Chiara Filippi $^{a}$ Silvia Tarentini ${ }^{a}$ Vania Marrone $^{a}$ Simonetta Monini ${ }^{a}$ \\ Annarita Vestrib ${ }^{b}$ Maurizio Barbara ${ }^{a}$

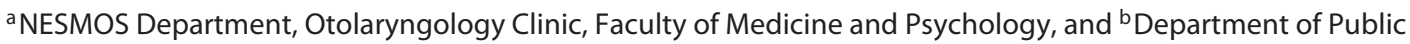 \\ Health and Infectious Diseases, Faculty of Medicine and Odontoiatrics, Sapienza University of Rome, Rome, Italy
}

\section{Keywords}

Ménière disease $\cdot$ Electrocochleography $\cdot$ Endolymphatic hydrops · Pressure treatment · Meniett device · Ventilation tube

\begin{abstract}
Objective: To identify eventual correlations between the effect of low-pressure treatment and endolymphatic hydrops in Ménière patients. Material and Methods: The study group consisted of subjects affected by definite Ménière disease (2015) and a severe degree of disability, who received a ventilation tube with or without a low-pressure treatment before undergoing a surgical procedure (vestibular neurectomy). After the placement of the ventilation tube, the subjects were either left alone with the tube or received 1 month of self-administered low-pressure therapy with a portable device. In all subjects, an electrocochleography (ECochG) was performed and specific questionnaires - Dizziness Handicap Inventory (DHI) and Functional Scale Level (FSL) - were completed before starting either arm of treatment, at the end of treatment, and then 3 and 6 months later. Results: All selected subjects presented with an ECochG pattern that was indicative of endolymphatic hydrops before starting either treatment. At the end of pressure treatment, $80 \%$ showed
\end{abstract}

\section{KARGER}

(C) 2017 S. Karger AG, Basel

E-Mail karger@karger.com

www.karger.com/aud symptomatic improvement while maintaining the hydropic ECochG pattern. At the 3-month control stage, the hydropic pattern resulted normalized $(<0.5)$ in all the improved subjects. Conclusions: Although 1 month of low-pressure treatment provided a positive symptomatological outcome, normalization of the hydropic ECochG parameters occurred only at a later time. Therefore, it is possible to assume that endolymphatic hydrops could be concurrent with a nonsymptomatic stage of Ménière disease, and that the anti-hydropic effect of the low-pressure treatment, if any, would present with a certain delay after its completion.

(c) 2017 S. Karger AG, Basel

\section{Introduction}

Ménière disease (MD) is a chronic, progressive, innerear disorder that is characterized by sudden attacks of vertigo with tinnitus, ear pressure, and low-tone fluctuating hearing loss [Lopez-Escamez et al., 2016]. During the disease course, the auditory and vestibular functions become progressively and, at times, independently affected regardless of the chosen treatment. Treatment options include a variety of medical approaches such as a low-salt diet, diuretics, steroids, calcium channel blockers, and va-

Maurizio Barbara, MD, PhD

NESMOS Department, Otolaryngology Clinic Sant'Andrea Hospital, Via di Grottarossa 1035 IT-00189 Rome (Italy)

E-Mail maurizio.barbara@uniroma1.it 
sodilators. Medical therapy is generally reported to provide disease control in nearly $70 \%$ of MD patients [Semaan and Megerian, 2011].

In those MD patients who are refractory to this preliminary approach, the intra-tympanic administration of different drugs (gentamicin, steroids) is used worldwide before considering a possible surgical solution. As well as intra-tympanic therapy, since the late 1990s a controlled, low-pressure treatment is also available through a portable device, built on the ground of a series of experimental studies that support its positive role for normalization of the inner ear fluid volumes [Tjernstrom, 1977; Densert et al., 1986]. This treatment may be considered conservative for the absence of cochlear damage as well as being noninvasive, only requiring the placement of a short-term ventilation tube (VT) for the direct transmission of pressure pulses through the external auditory canal into the middle ear. Despite not being very popular, several clinical reports have highlighted, in randomized trials, the positive role of such a device in selected cases of MD [Thomsen et al., 2005; Gürkov et al., 2012]. Fifteen years of continuous use at our centre has allowed us to confirm its positive role for the treatment of MD in patients who do not respond to initial medical therapies and who would thus be candidates for a surgical solution owing to the recurrence of disabling vertigo spells [Barbara et al., 2001, 2007]. A recent meta-analysis has reported that its use provided complete remission of the disease in $52 \%$ of the treated patients and substantial remission in $34 \%$ of them, with an overall positive effect in nearly $80 \%$ of cases [Zhang et al., 2016]. Nevertheless, the mechanism that underlies this positive effect remains unknown.

The present study was designed to shed some light on the possible relationships between local pressure treatment and endolymphatic hydrops, which is still considered the major landmark in MD and has been assessed by serial electrocochleographic (ECochG) recordings.

\section{Material and Methods}

Twenty consecutive patients ( 14 males and 6 females, mean age 56.3 years, range $41-83$ years), who presented in the last 2 years with a clinical picture of definite $\mathrm{MD}$, were included in this study. All patients had previously undergone unsuccessful medical therapy and were still complaining of invalidating and frequent vertigo spells (class D, American Academy of Otolaryngology-Head and Neck Surgery [AAO-HNS], 1995), so as to prompt the adoption of other invasive or destructive treatment approaches such as intratympanic gentamicin, sac surgery, or vestibular neurectomy. According to our pressure protocol [Barbara et al., 2001], they all first underwent trans-tympanic insertion of a VT. After 1 week, they were then randomized in one group that was left with the VT only (10 cases) and another group that was given 1 month of pressure therapy with the Meniett ${ }^{\circledR}$ device (Medtronic ENT, Jacksonville, FL, USA) (10 cases).

All subjects received a hearing evaluation that consisted of the assessment of their pure tone audiogram (PTA) and, more specifically, the mean threshold at 4 adjacent frequencies - 500, 1,000, 2,000 , and $4,000 \mathrm{~Hz}$ (PTA4) - that allowed the categorization of the patients, in accordance with the AAO-HNS criteria for hearing, into class I ( $<25 \mathrm{~dB})$, II (26-50 dB), III (51-70 dB), and IV ( $>71 \mathrm{~dB})$.

The first step of the protocol consisted of placing a transtympanic short-term VT under local anaesthesia. Soon after, an ECochG was performed inside an electrically and acoustically shielded room, using standard Otometrics equipment (ICS Chartr EP 200). With the patient in a supine position, a silver wire electrode, sheathed by a polyethylene tube embedded in hydrogel (Lilly TM-Wick electrode; Intelligent Hearing Systems, Miami, FL, USA), was positioned through the VT. The acoustic stimulation consisted of alternating polarity clicks (99-dB normal hearing level) that were presented at a rate of $7.1 / \mathrm{s}$, while the responses were averaged for 600 stimuli/s. The click-evoked summating potential (SP) amplitude was defined as the difference between the pre-stimulus baseline level and the first deflection. The action potential (AP) amplitude was measured from the baseline level to the first negative peak. After identifying both points, the SP/AP ratio was calculated. In this study, an SP/AP ratio of 0.50 or greater was considered indicative of a hydropic condition [Filipo et al., 1997]. Additionally, all subjects were asked to preliminarily complete the Dizziness Handicap Inventory (DHI) questionnaire for assessing their perceived disability, which allowed for investigation of the functional, emotional, and physical aspects; the higher the score (Q), the greater the perceived disability due to dizziness [Jacobson and Newman, 1990]. Vertigo control was also assessed according to the 1995 AAO-HNS classification, ranging from complete (grade A) to poor (grade D) control [Monsell, 1995], and by the Functional Scale Level (FSL).

The Meniett ${ }^{\circledR}$ device consists of a low-pressure generator that delivers $0.6-\mathrm{s}$ pressure pulses at $6 \mathrm{~Hz}$ within the range of $0-20 \mathrm{~cm}$ $\mathrm{H}_{2} \mathrm{O}$ to the ear canal. According to our protocol, each automatically set treatment cycle consisted of 3 sessions of 5 min each, including $1 \mathrm{~min}$ of pressure pulses and $40 \mathrm{~s}$ of pause. These daily sessions were prescribed to be performed during an entire month, starting 1 week after VT placement.

For the present protocol, the following 4 assessment times (T) were identified: the beginning Meniett treatment (T0, 1 week after VT placement); the end of Meniett treatment (T1, 30 days later); follow-up after 3 months from T0 (T2); and follow-up after 6 months from T0 (T3). The same times were also considered for assessing those subjects who only received the VT.

All the subjects signed an informed consent form, and the study was performed in accordance with the principles of the Declaration of Helsinki.

\section{Statistical Analysis}

Continuous data were summarized by mean and SD, while categorical data were summarized with count and percentage. Categorical variables were compared using the $\chi^{2}$ or Fisher exact tests. The $p$ values are 2 -sided; a $p$ value $\leq 0.05$ was considered statistically significant. All computations were carried out using SPSS version 21.0.

Meniett and Endolymphatic Hydrops

Audiol Neurotol 2017;22:24-29

DOI: $10.1159 / 000472245$ 


\section{Results}

The mean disease duration before entering the study was 3.2 years (range from 6 months to 25 years), and follow-up included up to 6 months after the end of treatment. During the first phase of the study (between T0 and T1), 2 subjects who were randomized for the VT only group asked to withdraw from it due to the persistence of debilitating symptoms and moved to the Meniett treatment (and group), which was in the subjects' opinion considered a therapeutic implementation. For ethical reasons their request was granted, although this produced an uneven number of subjects distributed in the 2 study groups (12 for the Meniett group vs. 8 for the VT group).

\section{T0}

An ECochG hydropic pattern was present in all subjects before they started the study. The mean SP/AP ratio

Table 1. Demographics of the study sample

\begin{tabular}{lll}
\hline & Meniett & VT \\
\hline Mean age, years & 56.9 & 55.3 \\
Male/female & $7 / 5$ & $7 / 1$ \\
Mean ECochG SP/AP & 0.7 & 0.7 \\
AAO-HNS class & D & D \\
DHI & 82.8 & 81.7 \\
Mean PTA4 & 62.6 & 59.7 \\
\hline
\end{tabular}

VT, ventilation tube; ECochG, electrocochleography; SP, summating potential; AP, action potential; AAO-HNS, American Academy of Otolaryngology-Head and Neck Surgery; DHI, Dizziness Handicap Inventory; PTA4, pure tone average at 500, 1,000, 2,000, and 4,000 Hz. value was 0.7 in the VT group (range $0.62-0.82$ ) and 0.7 in the Meniett group (range 0.53-0.9).

All the patients described a debilitating disease and were therefore classified as belonging to class $\mathrm{D}$, with a mean DHI score of 81.75 (range 66-92) for the VT group and 82.8 (range 64-100) for the Meniett group.

The mean PTA was $59.7 \mathrm{~dB}$ (min. 38.7; max. 71.2) in the VT group and $62.6 \mathrm{~dB}(\min .26 .2 \mathrm{~dB}$; $\max .82 .5 \mathrm{~dB})$ in the Meniett group. On the ground of the mentioned hearing thresholds, the patients were classified as stage I (no patient in either group), stage II (1 VT, 2 Meniett), stage III (4 VT, 7 Meniett), and stage IV (3 VT, 3 Meniett) (Table 1).

\section{T1}

At 1 month from treatment, an ECochG hydropic pattern was present in 9 patients (75\%) in the Meniett group and in all VT subjects (Table 2). In 3 patients in the Meniett group, the ECochG pattern appeared to decrease to a normal value range of $0.48,0.40$, and 0.42 , respectively. From a symptomatic point of view, 7 subjects $(58.4 \%)$ in the Meniett group and 2 VT subjects (25\%) had an improvement that was framed under class A or B (Table 2), as well as a mild DHI score $(<30)$. The mean PTA in the Meniett group was $59.6 \mathrm{~dB}$ (min. 16.2 dB; max. $82.5 \mathrm{~dB}$ ), dropping by $3 \mathrm{~dB}$; in the VT group, the mean PTA was $58.4 \mathrm{~dB}$ (min. 41.2; max. 73.7) with a drop of $1.3 \mathrm{~dB}$.

T2

Three months after T0, a hydropic SP/AP ratio was observed in 2 of the Meniett patients (16.7\%) and in 7 VT patients (87.5\%) (Table 2). All hydropic subjects in both groups had a DHI score $>50$, and they were still framed under class D. The mean PTA was $60.5 \mathrm{~dB}$ (min. $30 \mathrm{~dB}$;

Table 2. ECochG findings at the different follow-up times taken into consideration: before treatment (T0) and after 1 (T1), 3 (T2), and 6 months (T3) in the Meniett and VT groups

\begin{tabular}{|c|c|c|c|c|c|c|c|c|}
\hline & \multicolumn{2}{|l|}{ T0 } & \multicolumn{2}{|l|}{$\mathrm{T} 1$} & \multicolumn{2}{|l|}{$\mathrm{T} 2$} & \multicolumn{2}{|l|}{$\mathrm{T} 3$} \\
\hline & Meniett & VT & Meniett & VT & Meniett & VT & Meniett & $\mathrm{VT}^{\mathrm{a}}$ \\
\hline SP/AP >0.5 (hydrops) & $12(100)$ & $8(100)$ & $9(75)$ & $8(100)$ & $2(16.7)$ & $7(87.5)$ & $2(16.7)$ & $4(80)$ \\
\hline $\begin{array}{l}\text { SP/AP }<0.5 \text { (no } \\
\text { hydrops) }\end{array}$ & $0(0)$ & $0(0)$ & $3(25)$ & $0(0)$ & $10(83.3)$ & $1(12.5)$ & $10(83.3)$ & $1(20)$ \\
\hline
\end{tabular}

Values are $n(\%)$. ECochG, electrocochleography; VT, ventilation tube; SP/AP, difference between summating potential and action potential. ${ }^{\text {a }}$ Three subjects left the protocol after 3 months.

Covelli/Volpini/Atturo/Benincasa/Filippi/ Tarentini/Marrone/Monini/Vestri/Barbara 
max. $82.5 \mathrm{~dB}$ ) in the Meniett group (drop of $2.1 \mathrm{~dB}$ ) and $59.5 \mathrm{~dB}$ (min. 43.7, max. 72.5) in the VT group (drop of $0.2 \mathrm{~dB})$.

\section{T3}

Six months after T0, the 2 patients in the Meniett group who lacked symptom improvement, and instead experienced persistence of the hydropic ECochG pattern, were considered for vestibular neurectomy. Among the VT group, 3 subjects left the protocol after 3 months. Four out of the 5 remaining subjects continued to have a hydropic pattern with a disabling condition, while the non-hydropic VT subjects had symptom remission.

A significant difference in the hydropic pattern between the 2 study groups was found with the Fisher exact test at 3 months $(p=0.005)$ and 6 months $(p=0.028)$.

\section{Audiometric Results}

The mean PTA at the different follow-up steps is shown in Table 3. No differences were found in PTA before and 1, 3, and 6 months after Meniett treatment and in the VT group (T0-T1-T2-T3) [Thomsen et al., 2005]. No statistical difference in the pre- and post-treatment hearing thresholds was evidenced (Mann-Whitney U test and Friedman test).

Table 3. Mean PTA at the different follow-up times: before treatment (T0) and after 1 (T1), 3 (T2), and 6 months (T3) in the Meniett and VT groups

\begin{tabular}{lcccc}
\hline & T0 & T1 & T2 & T3 \\
\hline Meniett group & 62.6 & 59.6 & 60.5 & 62.7 \\
VT group & 59.7 & 58.4 & 59.5 & 58.1 \\
\hline
\end{tabular}

PTA, pure tone average; VT, ventilation tube.

\section{DHI Results}

The following 3 categories of severity could be distinguished: severe (score of 61-100), moderate (31-60), and mild (0-30). The single scores for each follow-up time are shown in Table 4. One month after treatment, 7 patients (58.4\%) reported a DHI improvement without normalization of the SP/AP ratio. At 3 and 6 months after treatment, a significant improvement at DHI corresponded to a remarkable reduction, with normalization, of the SP/AP ratio values (Table 4). Significant differences were found between Meniett and VT when testing the DHI at T2 (3 months after treatment) $(p=0.001)$ and T3 (6 months after treatment) $(p=0.005)$.

\section{Discussion}

Endolymphatic hydrops is considered the pathological finding of several inner ear disorders, including $\mathrm{MD}$ [Hallpike and Cairns, 1980]; as a result, its post-mortem finding allows defining MD as certain [Monsell, 1995]. Although promising reports have recently highlighted the possible role of magnetic resonance (MRI) techniques in obtaining a clear depiction of endolymphatic hydrops in living subjects [Wu et al., 2016], ECoch G has long been used for the same purpose taking into consideration the increased ratio between the SP and AP after stimulation with alternating polarity clicks [Gibson et al., 1977]. In particular, the elevation of the SP/AP ratio has been considered as proof for endolymphatic hydrops and explained by the displacement of the basilar membrane towards the scala tympani in response to endolymphatic overload [Eggermont, 1974].

To the best of our knowledge, no previous reports have been published on the possible relationships between $\mathrm{Me}$ niett treatment and endolymphatic hydrops, either based on imaging or electrophysiological techniques. There-

Table 4. DHI findings before treatment (T0) and after 1 (T1), 3 (T2), and 6 months (T3) in the Meniett and VT groups

\begin{tabular}{|c|c|c|c|c|c|c|c|c|}
\hline & \multicolumn{2}{|l|}{ T0 } & \multicolumn{2}{|l|}{$\mathrm{T} 1$} & \multicolumn{2}{|l|}{$\mathrm{T} 2$} & \multicolumn{2}{|l|}{$\mathrm{T} 3$} \\
\hline & Meniett & VT & Meniett & VT & Meniett & VT & Meniett & $\mathrm{VT}^{\mathrm{a}}$ \\
\hline Severe & $12(100)$ & $8(100)$ & $5(41.6)$ & $6(75)$ & $1(8.4)$ & $1(75)$ & $1(8.4)$ & $3(60)$ \\
\hline Moderate & $0(0)$ & $0(0)$ & $5(41.6)$ & $2(25)$ & $1(8.4)$ & $1(12.5)$ & $1(8.4)$ & $1(20)$ \\
\hline Mild & $0(0)$ & $0(0)$ & $2(16.8)$ & $0(0)$ & $10(83.2)$ & $1(12.5)$ & $10(83.2)$ & $1(20)$ \\
\hline
\end{tabular}

Values are $n$ (\%). DHI, Dizziness Handicap Inventory; VT, ventilation tube. ${ }^{\text {a }}$ Three subjects left the protocol after 3 months. 
fore, the present study was designed with this aim, and endolymphatic hydrops was assessed with ECochG. Although controversial reports have been published on the reliability of click-evoked ECochG [Gürkov et al., 2012], the use of this setting in the present study allowed for confirmation of our patients as classical MD subjects. In all of the patients, according to the protocol, pressure treatment was offered before proposing a surgical option in light of their disability and the recurrence of severe vertigo spells. In fact, it is our opinion that vertigo control should be the only reason for applying local pressure treatment, and that its eventual influence on the other MD symptoms (hearing loss, fullness, and tinnitus), although possible, should never represent its primary indication. In fact, when the main aim is resolution of nonvertigo symptoms, pressure treatment is likely to fail. Our suspicion, instead, is that its inappropriate indication, followed by failure, has contributed to raise some scepticism around its use. Another observation is related to the role of the presence of a VT, which has also been reported to provide a successful outcome in MD subjects [Montandon et al., 1988]. Consequently, for the present study it has been decided to include, via randomization, a group of Ménière subjects in whom VT insertion was not followed by pressure treatment, with the aim of ruling out any possible favourable role played by passive ventilation of a VT on MD. In this regard, the exit from the VT group of 2 subjects, who specifically asked to be included in the Meniett group due to aggravation of symptoms after insertion of the VT, consequently created an uneven distribution of the sample within the study group. Despite this methodological limitation, the findings evidenced in all the study phases, including the final follow-up observation time (T3), would in our opinion allow drawing some interesting remarks that are worth being discussed.

Once properly classified into class C or D, and selected for pressure treatment, it seemed interesting to determine whether endolymphatic hydrops was always underlying the symptomatic stage. To obtain this information, the ECochG examination was applied and used as a tool for monitoring the disease course immediately before and after treatment. All MD subjects included in this study presented with a pathological ECochG pattern that was compatible with endolymphatic hydrops. This first finding supports the validity of the sample selection, since in this preliminary phase of the study the clinical picture matched the electrophysiological finding, which is in favour of the presence of endolymphatic hydrops. However, after the first follow-up (T1), which was scheduled at the end of treatment, some subjects showed a mismatch between their symptoms and the electrophysiological findings. In fact, at stage T1, an abnormal, hydropic ECochG pattern was still present in a considerable number of subjects (75\%), although the majority of them (nearly $60 \%$ ) were already reporting a symptomatic improvement, shown by the drastic decrease in the DHI score and a shift of 1 or 2 classes. When looking at the results of stages T2 and T3, the symptomatic improvement that was found in $86 \%$ of the entire pressure-treated group corresponded to a normalization of the ECochG pattern, with an SP/AP ratio $<0.5$, while the subjects of the same group who showed no improvement ( 2 patients, equivalent to $12 \%$ of the group) still presented a hydropic ECochG pattern with persistence of a high DHI score and the same class of disease. This correlation between symptomatic control and the persistence of hydrops, which was also found in all subjects included in the VT group in the present study, seems extremely significant and emphasizes the importance of routine application of any diagnostic tool that would enable the acquisition of evidence of endolymphatic hydrops (ECochG or enhanced MRI). This concept has also been recently pointed out when analysing the negative outcome in subjects who underwent endolymphatic sac surgery [Liu et al, 2016].

Based on these findings, the active pressure treatment has been shown to be successful in the majority of the treated patients, which is in agreement with previously published studies; however, these studies lacked an explanation of the modality of action of the pressure device [Gürkov et al., 2012; Van Sonsbeek et al., 2015]. From the data collected in the present study, it would seem that Meniett therapy displays a positive influence on the distended endolymphatic space, as evidenced by the normalization of the SP/AP ratio in the responding subjects. In this regard, however, the ECochG pattern of behaviour seems controversial. In fact, the hydropic pattern remained stable at stage T1 (after 1 month of treatment) in the majority of subjects who were already showing a symptomatic improvement. Only 3 months later (stage T2) did the ECochG normalization correspond to the DHI decrease. One may argue that, from a symptomatological point of view, this finding could represent the effect of the natural course of the disease, but the evidence gathered in the control group seems to rule out this possibility. Hence, a positive outcome for the pressure treatment could also positively influence the inner ear fluid volume, but it may have a delayed effect that escapes in the first ECochG assessment at 1 month, while appearing and remaining stable at the 3 -month control. This delayed factor has in the past been already raised when re- 
ferring to the clinical expression of inner ear pathologies, basing on the histological finding of endolymphatic hydrops [Schuknecht, 1978]. It would therefore be plausible to speculate that the presence of partitions and valves within the membranous labyrinth could contribute to a sectorial behaviour of the different labyrinthine compartments when elicited by a hydropic condition, so that the pressure treatment would first positively influence the vestibular part, as shown by the early symptomatological improvement, and only at a later time the cochlear part, as assessed by the delayed electrophysiological findings. These considerations remain at a merely speculative level and obviously need to be substantiated by further conclusive studies.

The Meniett treatment has, in our hands, confirmed its positive value as a conservative treatment that can be offered to MD subjects who are refractory to medical therapy and for whom a destructive procedure, such as intra-tympanic gentamycin or vestibular neurectomy, could be indicated. Unlike gentamycin treatment, which may induce hearing deterioration, this pressure treatment is a true conservative option that does not affect hearing and is nearly devoid of possible complications, except for the need to tubulate the eardrum, which can result in permanent perforation. However, such a negative outcome has never been observed in over 100 subjects treated at our centre. At the same time, the presence of the VT tube was helpful in the present study because it allowed us to serially perform trans-tympanic ECochG with an exploration needle passing inside the lumen, therefore minimizing the patient's discomfort in this potentially invasive diagnostic procedure.

\section{Disclosure Statement}

The authors have no conflicts of interest to disclose.

\section{References}

Barbara M, Consagra C, Monini S, Nostro G, Harguindey A, Vestri A, Filipo R: Local pressure protocol, including Meniett, in the treatment of Ménière's disease: short-term results during the active stage. Acta Otolaryngol 2001; 121:939-944.

Barbara M, Monini S, Chiappini I, Filipo R: Meniett therapy may avoid vestibular neurectomy in disabling Meniere's disease. Acta Otolaryngol 2007;127:1136-1141.

Densert B, Densert O, Erlandsson B, Sheppard H: Transmission of complex pressure waves through the perilymphatic fluid in cats. Acta Otolaryngol 1986;102:403-409.

Eggermont JJ: Basic principles for electrocochleography. Acta Otolaryngol Suppl 1974; 316:75-84.

Filipo R, Cordier A, Barbara M, Bertoli GA: Electrocochleographic findings: Menière's disease versus sudden sensorineural hearing loss. Acta Otolaryngol Suppl 1997;526:21-23.

Gibson WP, Moffat DA, Ramsden RT: Clinical electrocochleography in the diagnosis and management of Meniere's disorder. Audiology 1977;16:389-401.

Gürkov R, Filipe Mingas LB, Rader T, Louza J, Olzowy B, Krause E: Effect of transtympanic low-pressure therapy in patients with unilateral Ménière's disease unresponsive to betahistine: a randomised, placebo-controlled, double-blinded, clinical trial. J Laryngol Otol 2012;126:356-362.
Hallpike CS, Cairns H: Observation on the pathology of Meniere's syndrome. J Laryngol Otol 1980;94:805-844.

Jacobson GP, Newman CW: The development of the Dizziness Handicap Inventory. Arch Otolaryngol Head Neck Surg 1990;116:424-427.

Liu IY, Sepahdari AR, Ishiyama G, Ishiyama A: High Resolution MRI shows presence of endolymphatic hydrops in patients still symptomatic after endolymphatic shunt surgery. Otol Neurotol 2016;37:1128-1130.

Lopez-Escamez JA, Carey J, Chung WH, Goebel JA, Magnusson M, Mandalà M, NewmanToker DE, Strupp M, Suzuki M, Trabalzini F, Bisdorff A: Diagnostic criteria for Menière's disease. Consensus document of the Bárány Society, the Japan Society for Equilibrium Research, the European Academy of Otology and Neurotology (EAONO), the American Academy of Otolaryngology-Head and Neck Surgery (AAO-HNS) and the Korean Balance Society (in Spanish). Acta Otorrinolaryngol Esp 2016;67:1-7.

Monsell EM: New and revised reporting guidelines from the Committee on Hearing and Equilibrium. American Academy of Otolaryngology-Head and Neck Surgery Foundation, Inc. Otolaryngol Head Neck Surg 1995; 113:176-178.

Montandon P, Guillemin P, Häusler R: Prevention of vertigo in Ménière's syndrome by means of transtympanic ventilation tubes. ORL J Otorhinolaryngol Relat Spec 1988;50: 377-381.
Semaan M, Megerian C: Meniere's disease: a challenging and relentless disorder. Otolaryngol Clin North Am 2011;44:383-403.

Schuknecht HF: Delayed endolymphatic hydrops. Otology Rhinol Laryngol 1978;87:743748.

Thomsen JC, Sass K, Odkvist L, Arlinger S: Local overpressure treatment reduces vestibular symptoms in patients with Menière's disease - secondary publication. A clinically randomised multicenter double-blind placebocontrolled study. Otol Neurotol 2005;26:6873.

Tjernstrom O: Effects of middle-ear pressure on the inner ear. Acta Otolaryngol 1977;83:1115.

Van Sonsbeek S, Pullens B, van Benthem PP: Positive pressure therapy for Ménière's disease or syndrome. Cochrane Database Syst Rev 2015; 10:3.

Wu Q, Dai C, Zhao M, Sha Y: The correlation between symptoms of definite Meniere's disease and endolymphatic hydrops visualized by magnetic resonance imaging. Laryngoscope 2016;126:974-979.

Zhang SL, Leng Y, Liu B, Shi H, Lu M, Kong WJ: Meniett therapy for Ménière's disease: an updated meta-analysis. Otol Neurotol 2016;37: 290-298. 